Article

\title{
Study on Properties Prediction and Braiding Optimization of Axial Braided Carbon/Carbon Composite
}

\author{
Chunguang Wang ${ }^{1, *}$, Peng Cao ${ }^{2}$, Min Tang ${ }^{3}$, Weiping Tian ${ }^{3}$, Ketong Liu ${ }^{4}$ and Baorui Liu ${ }^{5}$ \\ 1 College of Electrical and Control Engineering, Shaanxi University of Science \& Technology, \\ Xi'an 710021, China \\ 2 College of Architecture and Civil Engineering, Beijing University of Technology, Beijing 100124, China; \\ caopeng518888@126.com \\ 3 The Fourth Academy of CASC, Xi'an 710025, China; leegoop@126.com (M.T.); chencong0269@163.com (W.T.) \\ 4 College of Architecture and Civil Engineering, Xi'an University of Science and Technology, \\ Xi'an 710054, China; ketong-1982@163.com \\ 5 Science and Technology on Reliability and Environment Engineering Laboratory, Beijing Institute of \\ Structure and Environment Engineering, Beijing 100076, China; wolfman85@163.com \\ * Correspondence: wangcg1985@sust.edu.cn
}

Received: 29 February 2020; Accepted: 29 May 2020; Published: 5 June 2020

\begin{abstract}
It is well established that the microstructure has significant effects on the properties of axial braided $\mathrm{C} / \mathrm{C}$ composites. In this study, a method coupling the homogenization method and finite element method (FEM) was proposed to predict the relationship between the microstructure characteristics and macroscopic properties. Based on the representative volume element (RVE) model, the periodic displacement boundary condition was introduced to predict the equivalent elastic properties of the RVE and component of C/C composite material, and the coefficient of thermal expansion (CTE) of the material was predicted by the energy prediction method. The predicted results were in good agreement with experimental results. By predicting the thermal and mechanical properties of the materials with different braiding spacing and fiber rod diameter, the variation of the properties with braiding spacing and fiber rod diameter was obtained. The research methods and results in this paper could provide important references for the optimization and rational application of composite materials.
\end{abstract}

Keywords: axial braided C/C composites; macroscopic properties; prediction method; microstructure characteristics; braiding spacing

\section{Introduction}

Axial braided C/C composite is a special carbon fiber reinforced carbon matrix composite. Both the reinforcing phase and matrix phase are composed of pure carbon [1-5]. It exhibits many superior properties, such as high specific strength, good thermal stability, wear resistance and ablative resistance. Specially, different from common materials, its mechanical properties do not decrease but rather increase with the increase of temperature, which makes it widely used in aviation and aerospace fields [6,7]. Due to its complex internal microstructures, such as porosity, cracks, pore mean and pore shape, it is difficult to quantify the effects of microstructure characteristics on its macroscopic properties based on experiments. Therefore, numerical and theoretical methods calibrated and verified by experiments are required to evaluate the relationship between its microstructure characteristics and macro properties [8-12]. 
Many methods have been introduced to predict the mechanical properties of braided $\mathrm{C} / \mathrm{C}$ composites [13-16]. Among them, the stiffness performance has been well investigated. With the development of finite element method (FEM), the method for predicting the properties based on the homogenization theory provides a new idea for predicting the properties of $\mathrm{C} / \mathrm{C}$ composite materials. Because the FEM is used in this method, the microstructure characteristics and distribution in the fiber rod (bundle) can be considered. Because the homogenization theory is combined, the interaction between components can be taken into account in the model, so that the stiffness prediction accuracy of C/C composite materials is higher [17-19]. The energy method-based prediction method of CTE of composite materials derived by Zhang [20-23] is more convenient to calculate than the homogenization method, and has been gradually popularized in the industry.

In this paper, the braided structure characteristics of axial braided $\mathrm{C} / \mathrm{C}$ composites were considered, the homogenization method was combined with FEM by ABAQUS. Besides, the energy method was introduced to establish the prediction method of macroscopic properties of materials. A Python program was used to generate the matrix, interface and fiber rod (bundle) geometric models that meet the distribution law of microstructure characteristics and predict their stiffness. The predicted results and the measured results were taken as the input of FEM. Based on the RVE model of materials, periodic displacement boundary conditions were introduced to predict the equivalent elastic properties and the CTE of axial braided C/C composites [17-20]. By predicting the macroscopic properties of the material with different braiding spacing and fiber rod diameter, the variation of material properties was obtained, which provided a reference for the optimization and rational application of the material.

\section{A Method for Predicting the Macroscopic Properties of Axial Braided C/C Composite}

\subsection{Homogenization Process and Stiffness Prediction Method}

The homogenization theory has been widely recognized to predict the stiffness of composites, and its concept is shown in Figure 1. In order to solve the difficulty of large-scale macro-micro joint computation, the concept of microelement is introduced. A microelement represents a point in the composite structure, and its average stress and strain relation is regarded as the effective constitutive relation of composite. In fact, the non-uniform element is replaced by a representative volume element (RVE) with the above average stress and strain relationship. The above concepts are applied to every point of the composite structure. Based on such way, the original problem of analyzing the non-uniform composite structure was converted to the problem of analyzing the properties of the macro structure after homogenization, which greatly simplified the original problem [24,25].

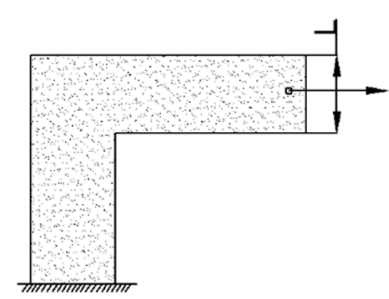

(a)

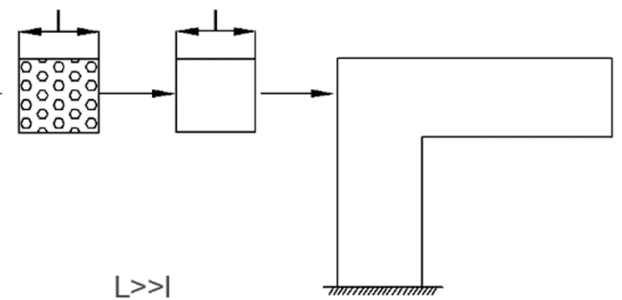

(c)

(d)

Figure 1. Composite structure, representative volume element and equivalent structure. (a) original structure (b) micro element (c) homogenized element (d) homogenized structure.

Meso-level composites often exhibit a high degree of heterogeneity, and even in the neighborhood $\varepsilon$ with a very small macroscopic position $x$, the field variables will vary greatly, as shown in Figure 2. 


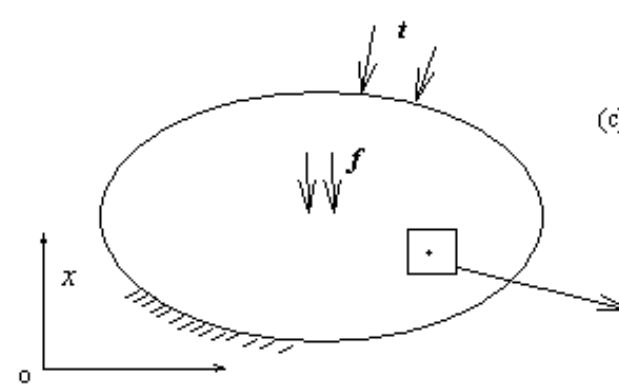

(a)

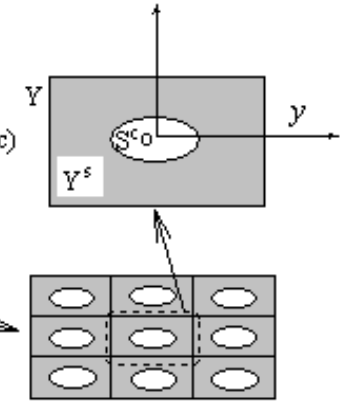

(b)

Figure 2. Composite material with mesoscopic structure. (a) Macro structure (b) Mesoscopic structure and its periodicity (c) RVE.

Considering the periodicity of the composite structure, these field variables can be expressed as follows:

$$
\Phi^{\varepsilon}(x)=\Phi(x, y+k Y), y=x / \varepsilon, \frac{\partial \phi^{\varepsilon}}{\partial x}=\frac{\partial \phi}{\partial x}+\frac{1}{\varepsilon} \frac{\partial \phi}{\partial y}
$$

where, $x$ is the macroscopic scale, $y$ is the mesoscopic scale, $\varepsilon$ is the ratio of the two scales, $Y$ is the period of the periodic function, and $k$ is the integer.

The displacement is expressed as a function of double-scale coordinates and expanded according to small parameters:

$$
u^{\varepsilon}(x)=(x, y)+\varepsilon^{1} u^{1}(x, y)+\varepsilon^{2} u^{2}(x, y)+\cdots, y=x / \varepsilon
$$

$u^{(i)}(x, y)$ is a periodic function of $Y$. According to constitutive equation and geometric equation in elastic mechanics, the above asymptotic expansion was substituted into the equilibrium equation, and the left side of the equation was arranged as an asymptotic expansion with respect to $\varepsilon$. We set the coefficient of $\varepsilon^{i}(i=-1,0,1, \cdots)$ as zero to obtain a series of governing equations, in which:

$$
\begin{gathered}
\frac{\partial \sigma_{i j}^{(-1)}(x, y)}{\partial y_{j}}=0 \\
\frac{\partial \sigma_{i j}^{(0)}(x, y)}{\partial y_{j}}+\frac{\partial \sigma_{i j}^{(-1)}(x, y)}{\partial x_{j}}=0
\end{gathered}
$$

In Equation (4), $\sigma_{i j}(x, y)$ is a function of stress field. By simplifying the operation, the following equation was obtained:

$$
\begin{gathered}
\sigma_{i j}^{(0)}=\left(C_{i j k l}-C_{i j m n} \partial \chi_{m}^{k l} / \partial y_{n}\right) \varepsilon_{x k l}\left(u^{(0)}\right) \\
{\left[C_{i j k l}\left(\chi_{\left(k, y_{l}\right) m n}+I_{k l m n}\right)\right]_{y_{j}}=0 \text { on } Y} \\
\chi_{i m n}(\mathrm{y})=\chi_{i m n}(\mathrm{y}+Y) \text { on } \partial Y \\
\chi_{i m n}(\mathrm{y})=0 \text { on } \partial Y^{\text {vert }}
\end{gathered}
$$

Among them:

$$
\begin{gathered}
I_{k l m n}=\left(\delta_{m k} \delta_{n l}+\delta_{n k} \delta_{m l}\right) / 2 \\
\chi_{\left(k, y_{l}\right) m n}=\frac{1}{2}\left(\frac{\partial \chi_{k m n}}{\partial y_{l}}+\frac{\partial \chi_{l m n}}{\partial y_{k}}\right)
\end{gathered}
$$


The periodic function $Y$ can be expressed as the region of a single cell in the axial braided carbon/carbon composite. $\partial Y$ is the boundary of the cell, and $\partial Y^{\mathrm{vert}}$ is the vertex of the cell on the boundary. The homogenization coefficient of a single cell can be expressed as:

$$
C_{i j k l}^{H}=\frac{1}{|Y|} \int_{Y} C_{i j k l}\left(\chi_{\left(k, y_{l}\right) m n}+I_{k l m n}\right) d V
$$

$\sigma^{(0)}(x, y)$ is the second term of the asymptotic expansion of $\sigma^{\varepsilon}(x, y)$, which is called the mesoscopic stress field. The mesoscopic stress field includes the macroscopic parameters $x$ and the mesoscopic parameters $y$, which reflect the fluctuation of stress in the scale of RVE.

Gauss theorem is used to treat the governing equation of the homogenization coefficient of Equation (11), and the equation is converted into a "weak" solution equivalent to the original problem as follows:

$$
\int_{Y} C_{i j k l} \chi_{\left(k, y_{l}\right) m n} \frac{1}{2}\left(\frac{\partial \delta v_{i}}{\partial y_{j}}+\frac{\partial \delta v_{j}}{\partial y_{i}}\right) d V=-\sum_{p} \int_{\partial Y^{p}-\partial Y^{p} \cap \partial Y} C_{i j m n} n_{j} \delta v_{i} d S
$$

From Equation (12) can be found in the original control equation is equivalent to the three dimensional linear elastic problems in the form of the "weak" solution of equation, the difference is that the material within a plane distribution related to the material properties and boundary shape, the plane distribution of force on the internal boundary of different material in the RVE $\left(\partial Y^{p}-\partial Y^{p} \cap \partial Y\right)$, pointing to the internal material area, the direction of the $i$ component for $C_{i j m n} n_{j}$.

The two terms in the integral sign of Equation (12) were separated to obtain the deformation of the homogenization coefficient:

$$
C_{i j k l}^{H}=\frac{1}{|Y|} \int_{Y} C_{i j k l} \chi_{\left(k, y_{l}\right) m n} d V+\frac{1}{|Y|} \int_{Y} C_{i j m n} d V
$$

From Equation (13), it can be seen that in the boundary force method, the homogenization coefficient is equal to the volume average of the stress of the three-dimensional linear elastic problem on the RVE plus the volume average of the elastic tensor on the RVE [16-18]. The homogenization coefficient is the effective elastic property of macroscopic structure.

\subsection{A Method for Predicting CTE of Composites Based on Energy Method}

The CTE prediction method based on the energy method is more convenient to calculate than the simple homogenization method. Therefore, the following method combined with FEM was used to calculate the CTE of materials in this paper [19,22].

The basic idea of the energy method is that based on the relationship between microstructure and homogeneous equivalent body, the energy expression of the equivalent property of composite materials can be obtained by deducing the relationship between the equivalent property of composite materials and the deformation energy of microstructure [16,17].

For the microstructure shown in Figure 1, when the temperature rise value is $\Delta T$, the average stress and average strain of the microstructure have the following relationship:

$$
\bar{\sigma}=C^{H}\left(\bar{\varepsilon}-\alpha^{H} \Delta T\right)
$$

where $\mathrm{C}^{H}, \alpha^{H}=\left[\alpha_{11}^{H}, \alpha_{22}^{H}, \alpha_{33}^{H}\right]$ are the stiffness matrix and CTE matrix of axial braided C/C composites.

The deformation energy of the microstructure of the material is shown in Equation (15):

$$
C=\int_{\Omega} \varepsilon^{T} D \varepsilon d \Omega
$$

For working condition $1\left(u_{2}=u_{3}=0\right)$ as shown in Figure 3a, the microstructure deformation energy caused by $\Delta T$ temperature change is the same as that of working condition 4 without temperature 
load as shown in Figure $3 \mathrm{~b}$. The displacement load $u_{1}$ in working condition 4 is equal to the displacement load under working condition 1 . The force $f$ is the interface force caused by the difference of thermal stress between the two loads. The expressions of the two are Equations (16) and (17).

$$
\begin{gathered}
u_{1}=\left(\alpha_{11}^{H} \Delta T+\left(D_{1122}^{H} / D_{1111}^{H}\right) \alpha_{22}^{H} \Delta T+\left(D_{1133}^{H} / D_{1111}^{H}\right) \alpha_{33}^{H}\right) l \\
f=\int_{s}\left[D\left(E_{1}\right) \alpha\left(\alpha_{1}\right)-D\left(E_{2}\right) \alpha\left(\alpha_{2}\right)\right] \Delta T d_{s}
\end{gathered}
$$

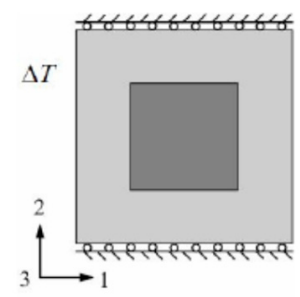

(a) Condition 1
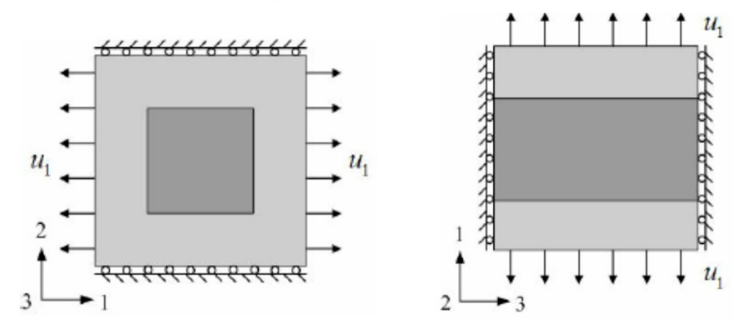

(c) Condition 5
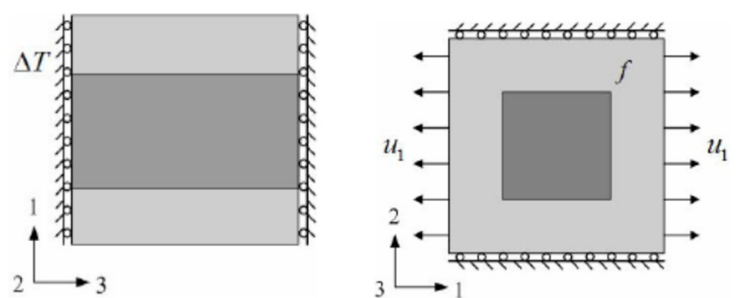

(b) Condition 4
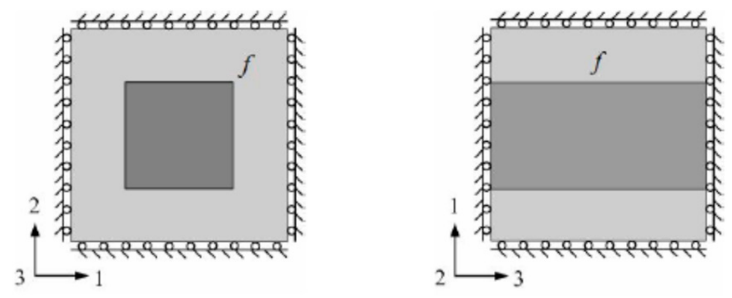

(d) Condition 6

Figure 3. Microstructure forms under different operating conditions. Among of them, working condition 2 and working condition 3 are similar to working condition 1, which are not completely shown in the figure.

For the microstructure of working condition 1, the variable properties of its homogeneous equivalent are equal to the difference between the variable properties of working condition 1 and that of working condition 6, as shown in Equation (18):

$$
C_{e m}^{(1)}=C_{m}^{(1)}-C_{m}^{(6)}=D_{1111}^{H}\left(\alpha_{11}^{H} \Delta T+\left(D_{1122}^{H} / D_{1111}^{H}\right) \alpha_{22}^{H} \Delta T+\left(D_{1133}^{H} / D_{1111}^{H}\right) \alpha_{33}^{H} \Delta T V\right)^{2}
$$

Similar to Equation (18), the variable properties of the homogeneous equivalent of condition 2 $\left(u_{1}=u_{3}=0\right)$ and condition $3\left(u_{1}=u_{2}=0\right)$ can be expressed as Equations (19) and (20):

$$
\begin{aligned}
& C_{e m}^{(2)}=C_{m}^{(2)}-C_{m}^{(6)}=D_{2222}^{H}\left(\alpha_{22}^{H} \Delta T+\left(D_{1122}^{H} / D_{2222}^{H}\right) \alpha_{11}^{H} \Delta T+\left(D_{2233}^{H} / D_{2222}^{H}\right) \alpha_{33}^{H} \Delta T\right)^{2} \\
& C_{e m}^{(3)}=C_{m}^{(3)}-C_{m}^{(6)}=D_{3333}^{H}\left(\alpha_{33}^{H} \Delta T+\left(D_{1133}^{H} / D_{3333}^{H}\right) \alpha_{11}^{H} \Delta T+\left(D_{2233}^{H} / D_{3333}^{H}\right) \alpha_{22}^{H} \Delta T\right)^{2}
\end{aligned}
$$

The FEM equation of the microstructure under operating conditions 4, 5 and 6 can be expressed as Equation (20).

$$
\left[\begin{array}{ll}
k_{11} & k_{12} \\
k_{21} & k_{22}
\end{array}\right]\left[\begin{array}{l}
U_{1}^{(i)} \\
U_{2}^{(i)}
\end{array}\right]=\left[\begin{array}{l}
F_{1}^{(i)} \\
F_{2}^{(i)}
\end{array}\right] i=4,5,6
$$


where, $U_{1}^{(i)}$ is the unknown node displacement vector under condition $i$, and $U_{2}^{(i)}$ is the known node displacement vector. $F_{1}^{(i)}$ is the known node force vector under condition $i$, and $F_{2}^{(i)}$ is the unknown node force vector. To solve Equation (20), the following Equation (21) can be obtained.

$$
\begin{gathered}
U_{1}^{(i)}=K_{11}^{-1} F_{1}^{(i)}-K_{11}^{-1} K_{12} U_{2}^{(i)} \\
F_{2}^{(i)}=K_{21} K_{11}^{-1} F_{1}^{(i)}+\left(K_{22}-K_{21} K_{11}^{-1} K_{12}\right) U_{2}^{i}
\end{gathered}
$$

The microstructural variable performance of working condition $i$ can be expressed in the following form:

$$
C_{m}^{(i)}=\int_{\Omega} \varepsilon^{(i) T} D \varepsilon^{(i)} d_{\Omega}=F_{1}^{(i) T} U_{1}^{(i)}+F_{2}^{(i) T} U_{2}^{(i)}
$$

The calculation method of CTE of microstructure can be obtained by solving Equation (22) as shown in Equation (23):

$$
\begin{aligned}
& \alpha_{11}^{H}=\frac{\left(\frac{D_{1122}^{H}}{D_{111}^{H}} \frac{D_{2233}^{H}}{D_{222}^{H}}-\frac{D_{1133}^{H}}{D_{1111}^{H}}\right)\left(\frac{D_{2233}^{H}}{D_{333}^{H}} Q-R\right)-\left(\frac{D_{2233}^{H}}{D_{3333}^{H}} \frac{D_{2233}^{H}}{D_{2222}^{H}}-1\right)\left(\frac{D_{1122}^{H}}{D_{1111}^{H}} Q-P\right)}{\left(\frac{D_{1112}^{H} D_{2233}^{H}}{D_{1111}^{H}} \frac{D_{2222}^{H}}{D_{113}^{H}}-\frac{D_{133}^{H}}{D_{111}^{H}}\right)\left(\frac{D_{2233}^{H}}{D_{3333}^{H}} \frac{D_{1122}^{H}}{D_{2222}^{H}}-\frac{D_{1333}^{H}}{D_{3333}^{H}}\right)-\left(\frac{D_{233}^{H}}{D_{3333}^{H}} \frac{D_{2233}^{H}}{D_{2222}^{H}}-1\right)\left(\frac{D_{122}^{H}}{D_{1111}^{H}} \frac{D_{1122}^{H}}{D_{2222}^{H}}-1\right)} \\
& \alpha_{22}^{H}=\frac{\left(\frac{D_{1122}^{H}}{D_{222}^{H}} \frac{D_{1133}^{H}}{D_{111}^{H}}-\frac{D_{2233}^{H}}{D_{2222}^{H}}\right)\left(\frac{D_{1133}^{H}}{D_{3333}^{H}} P-R\right)-\left(\frac{D_{1133}^{H}}{D_{3333}^{H}} \frac{D_{1133}^{H}}{D_{1111}^{H}}-1\right)\left(\frac{D_{1122}^{H}}{D_{2222}^{H}} P-Q\right)}{\left(\frac{D_{1112}^{H}}{D_{2222}^{H}} \frac{D_{1133}^{H}}{D_{1111}^{H}}-\frac{D_{2233}^{H}}{D_{2222}^{H}}\right)\left(\frac{D_{1133}^{H}}{D_{3333}^{H}} \frac{D_{1122}^{H}}{D_{1111}^{H}}-\frac{D_{2333}^{H}}{D_{3333}^{H}}\right)-\left(\frac{D_{133}^{H}}{D_{3333}^{H}} \frac{D_{1133}^{H}}{D_{1111}^{H}}-1\right)\left(\frac{D_{1122}^{H}}{D_{2222}^{H}} \frac{D_{1122}^{H}}{D_{1111}^{H}}-1\right)} \\
& \alpha_{33}^{H}=\frac{\left.\left(\frac{D_{1133}^{H}}{D_{333}^{H}} \frac{D_{1122}^{H}}{D_{1111}^{H}}-\frac{D_{2233}^{H}}{D_{333}^{H}}\right)\left(\frac{D_{2233}^{H}}{D_{333}^{H}} P-Q\right)-\left(\frac{D_{1122}^{H}}{D_{2222}^{H}} \frac{D_{1122}^{H}}{D_{1111}^{H}}\right)-1\right)\left(\frac{D_{1133}^{H}}{D_{3333}^{H}} P-R\right)}{\left.\left(\frac{D_{1133}^{H}}{D_{3333}^{H}} \frac{D_{1222}^{H}}{D_{1111}^{H}}-\frac{D_{22233}^{H}}{D_{3333}^{H}}\right)\left(\frac{D_{1112}^{H}}{D_{2222}^{H}} \frac{D_{1133}^{H}}{D_{1111}^{H}}-\frac{D_{2233}^{H}}{D_{2222}^{H}}\right)-\left(\frac{D_{1122}^{H}}{D_{2222}^{H}} \frac{D_{1122}^{H}}{D_{1111}^{H}}\right)-1\right)\left(\frac{D_{1133}^{H}}{D_{3333}^{H}} \frac{D_{133}^{H}}{D_{1111}^{H}}-1\right)}
\end{aligned}
$$

In the equation $P=\sqrt{\frac{C_{m}^{(1)}-C_{m}^{(6)}}{D_{1111}^{H}(\Delta T)^{2} V}}, Q=\sqrt{\frac{C_{m}^{(2)}-C_{m}^{(6)}}{D_{2222}^{H}(\Delta T)^{2} V}}, R=\sqrt{\frac{C_{m}^{(3)}-C_{m}^{(6)}}{D_{3333}^{H}(\Delta T)^{2} V}}$.

\subsection{The Boundary Conditions}

According to the braided characteristics of the axial braided $\mathrm{C} / \mathrm{C}$ composites studied in this paper, each surface of the RVE model must be set as a periodic symmetric boundary condition. The displacement of the RVE should also meet the periodic boundary conditions [17]. For the periodic RVE structure with spatial translational symmetry, the periodic condition of its displacement can be expressed as follows:

$$
\begin{aligned}
& u_{2}\left(y_{1}^{0}, y_{2}, y_{3}\right)=u_{1}\left(y_{1}^{0}+Y_{1}, y_{2}, y_{3}\right) \\
& u_{2}\left(y_{1}, y_{2}^{0}, y_{3}\right)=u_{1}\left(y_{1}, y_{2}^{0}+Y_{2}, y_{3}\right) \\
& u_{2}\left(y_{1}, y_{2}, y_{3}^{0}\right)=u_{1}\left(y_{1}, y_{2}, y_{3}^{0}+Y_{3}\right)
\end{aligned}
$$

\section{The Introduction of the Experiment Carried out in the Early Stage}

\subsection{Properties Test of Fiber Rod}

Under the premise of minimum damage, the z-direction fiber rod (XY direction is transverse) was stripped, and the tensile and compression properties of the fiber rod were tested to obtain its stiffness properties. A special fixture was designed for its test. In the test, the unconstrained connection mechanism was adopted to eliminate the error caused by eccentricity when the sample was loaded. The accurate strain of fiber rods was obtained by non-contact optical strain testing system (ARAMIS, 2.3M, GOM, Brunswick, Germany). The obtained stiffness properties of the fiber rod are shown in Table 1. 
Table 1. Stiffness characteristics of fiber rod.

\begin{tabular}{ccccc}
\hline \multirow{2}{*}{ Item } & \multicolumn{2}{c}{$E_{x}, \mathrm{GPa}$} & \multicolumn{2}{c}{$v_{x y}=v_{x z}$} \\
\cline { 2 - 5 } & Mean Value & Standard Deviation & Mean Value & Standard Deviation \\
\hline Tension & 211.1 & 10.3 & 0.06 & 0.02 \\
Compression & 99.4 & 8.47 & 0.15 & 0.03 \\
\hline
\end{tabular}

Note: Angle mark $x$ represents the fiber rod axial direction, Angle mark $y$ and $z$ represent the transverse direction.

\subsection{Macroscopic Properties Test}

The macroscopic mechanical properties of axial braided C/C composites mainly include uniaxial tensile test and uniaxial compression test. Samples under different loading conditions were designed according to the braiding mode and product size of the axial braided C/C composite. Samples with different directions including fiber rod direction (axial direction) and fiber bundle direction (radial direction) were tested and more than 10 samples were prepared for each group. The exact strain of specimens was obtained by non-contact optical strain testing system (ARAMIS). The stiffness properties of axial braided C/C composites are shown in Table 2.

Table 2. Stiffness characteristics of axial braided C/C composites.

\begin{tabular}{ccccccccc}
\hline \multirow{2}{*}{ Item } & \multicolumn{2}{c}{$E_{X}, \mathrm{GPa}\left(G_{X Y}, \mathrm{GPa}\right)$} & \multicolumn{2}{c}{$\boldsymbol{v}_{\boldsymbol{X}}$} & \multicolumn{2}{c}{$E_{Z}, \mathrm{GPa}\left(G_{X Z}, \mathrm{GPa}\right)$} & \multicolumn{2}{c}{$\boldsymbol{v}_{X Z}$} \\
\cline { 2 - 9 } & $\begin{array}{c}\text { Mean } \\
\text { Value }\end{array}$ & $\begin{array}{c}\text { Standard } \\
\text { Deviation }\end{array}$ & $\begin{array}{c}\text { Mean } \\
\text { Value }\end{array}$ & $\begin{array}{c}\text { Standard } \\
\text { Deviation }\end{array}$ & $\begin{array}{c}\text { Mean } \\
\text { Value }\end{array}$ & $\begin{array}{c}\text { Standard } \\
\text { Deviation }\end{array}$ & $\begin{array}{c}\text { Mean } \\
\text { Value }\end{array}$ & $\begin{array}{c}\text { Standard } \\
\text { Deviation }\end{array}$ \\
\hline Tension & 38.3 & 2.3 & 0.28 & 0.41 & 40.5 & 4.2 & 0.09 & 0.05 \\
Compression & 20.9 & 1.9 & 0.28 & 0.04 & 22.1 & 2.1 & 0.09 & 0.05 \\
Shear & 12.6 & 1.3 & & & 3.4 & 0.5 & & \\
\hline
\end{tabular}

Note: Angle mark $Z$ represents the fiber rod axial direction, Angle mark $X$ and $Y$ represent the transverse direction.

\section{RVE Establishment and Properties of Component}

\subsection{RVE Establishment}

The RVE of axial braided C/C composite material is a rectangular structure composed of fiber rod, fiber bundle, matrix and interface. The braided structure and phase scale of each component are shown in Figure 4. Thin carbon fiber rigid rod formed by pultrusion forms the axial reinforcing network, and the soft carbon fiber bundle is woven into the pre-texture. The fiber rods are arranged in an equilateral triangle in the axial direction, and the fiber bundles are successively increased through the $0^{\circ}, 60^{\circ}$ and $120^{\circ}$ channels of cambium formed by the fiber rods, and so on until the required size of pre-texture is formed. High density 4D C/C composites were prepared by asphalt impregnation, carbonization, densification and high temperature treatment. The minimum unit of this pre-texture is axially symmetric, and its braided thickness accumulates axially, so it is called axial braided $\mathrm{C} / \mathrm{C}$ composite. 

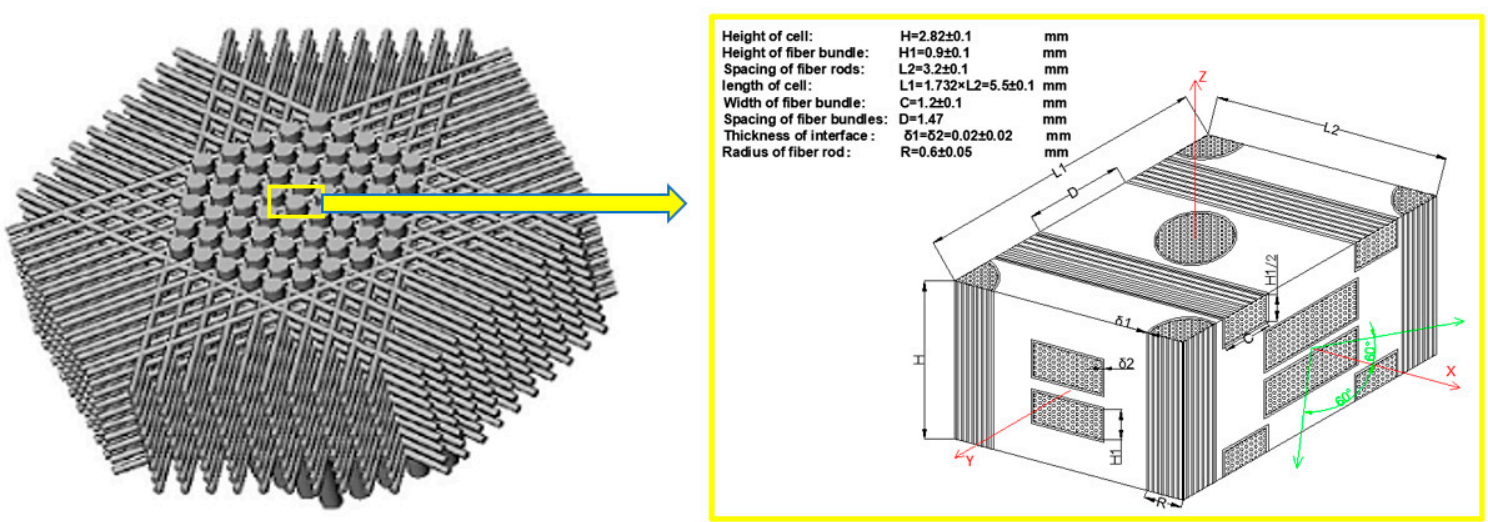

Figure 4. RVE of axial braided C/C composite.

The generated finite element model of axial braided $\mathrm{C} / \mathrm{C}$ composite material composed from the RVE model is shown in Figure 5. The division of the grid is highly related to the application of periodic boundary conditions, so it is necessary to strictly guarantee the uniformity of the grid nodes on the corresponding surface of RVE. In this paper, C3D8 elements and C3D6 elements were used to mesh the fiber rod, fiber bundle and matrix. The fiber rod/matrix interface and fiber bundle/matrix interface were all meshed by C3D8 elements [24-28].

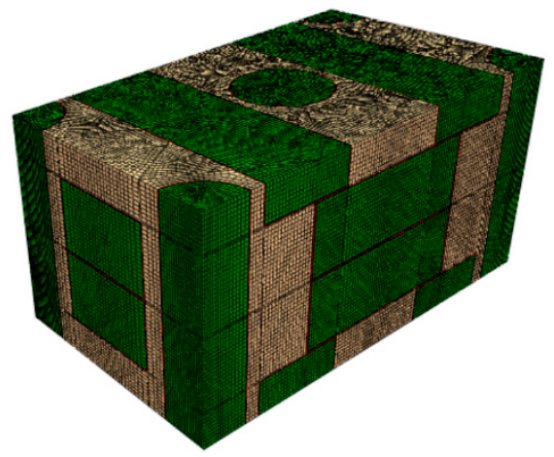

(a) Finite element model of RVE

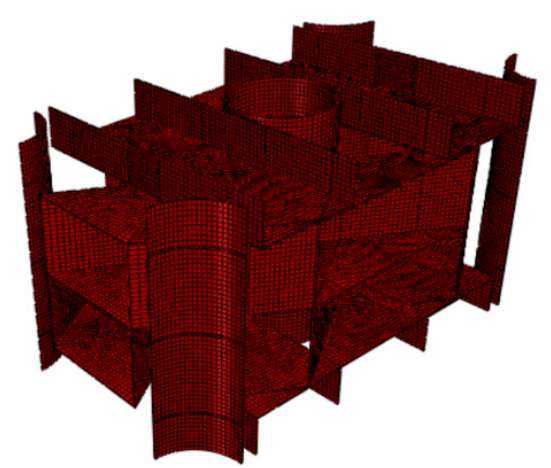

(b) The interface element

Figure 5. Finite element model of axial braided C/C composite material.

\subsection{Study on the Properties of Components}

The premise of RVE model performance calculation with finite element technique is that the properties of each meso components (including the fiber rod and bundle, matrix and interface) are known. Due to the poor stripping and testability of the meso components, some of the necessary properties need to be obtained in combination with numerical calculations.

\subsubsection{Mechanical Properties of Matrix and Interface}

The pores and cracks in the matrix and interface of the axial braided C/C composite were regarded as inclusions with different shapes and sizes and introduced into the pure carbon matrix. In this paper, the random geometric models meeting objective distribution law of microstructure characteristics were generated by a subroutine coded by python and its mechanical properties were calculated by FEM, which can consider the interaction between various microstructures [28-32].

In order not only to simplify the calculation workload but also to contain sufficient pore information, the side length of the calculation model of the matrix was selected as $0.4 \mathrm{~mm}$, and the side length of the interface was selected as $0.02 \mathrm{~mm}$, and the generated model was shown in Figure 6. In the model, the pores are mainly ellipsoid, and the ratio of long axis and short axis of ellipsoid at some locations is 
large, resulting in cracks. In the early stage, the non-pore matrix modulus was 12.34 GPa and Poisson's ratio was 0.23 by using nano-indentation experiment [16,17], which was used as the material input for finite element calculation.

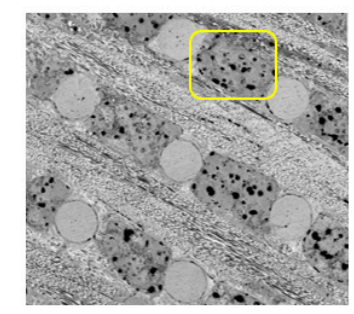

(a) Microstructure of matrix pores

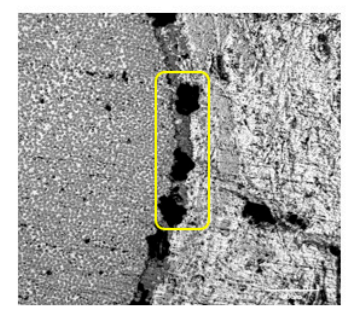

(b) Microstructure of interfacial pores

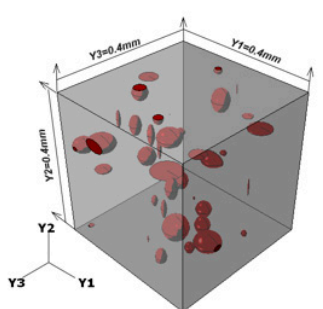

(c) Matrix stochastic model

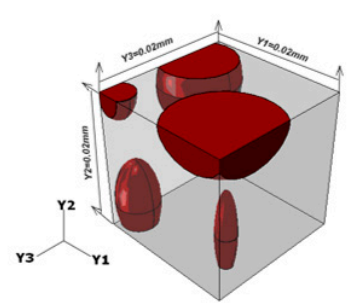

(d) Interfacial stochastic model

Figure 6. Computational model of matrix and interface.

Figure 7a shows the effect of porosity on the elastic properties of the matrix. It can be seen that the elastic properties of the matrix vary linearly with porosity. The increase of porosity leads to the decrease of both elastic modulus and Poisson's ratio.

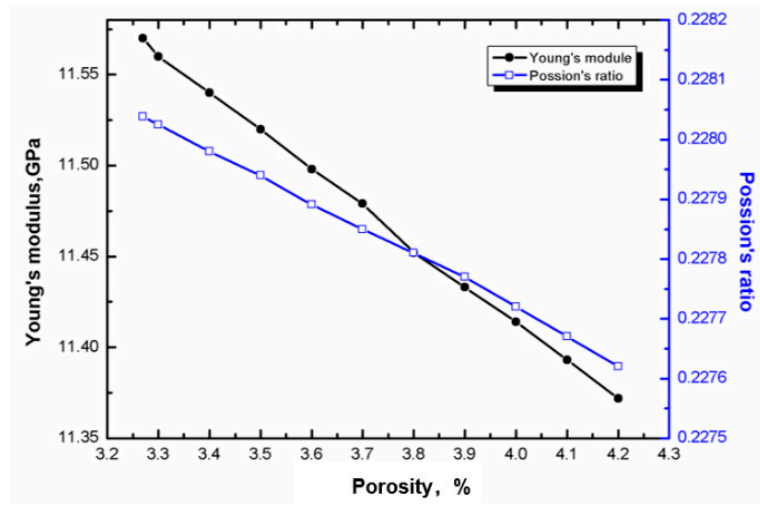

(a) Effect of porosity on the elastic properties of matrix

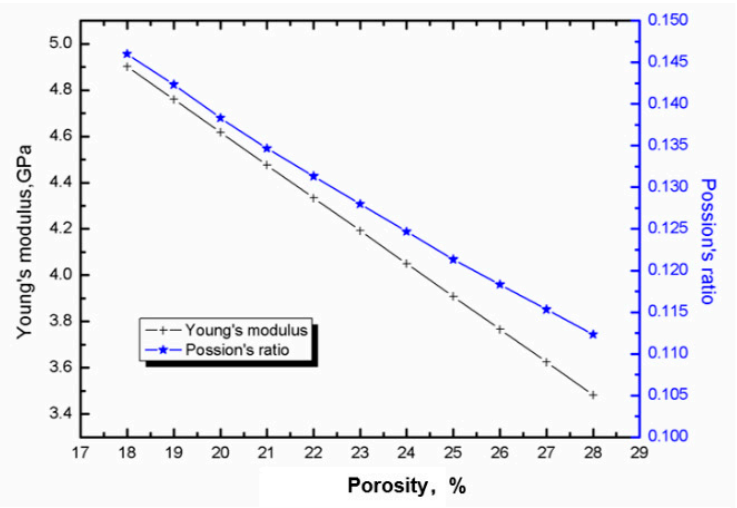

(b) Effects of porosity on the elastic properties of interface

Figure 7. Effect of porosity on the elastic properties of matrix and interface.

Figure $7 \mathrm{~b}$ shows the influence of porosity on the interface elastic properties. It can be found that the influence trend of porosity on the interface elastic properties is the same as that of porosity on the matrix.

Based on the statistical study in [2], the porosity of the matrix was 0.036 , and the modulus and Poisson's ratio were $11.5 \mathrm{GPa} / 0.228$ respectively, obtained from Figure $7 \mathrm{a}$. Based on the statistical study in [2], the porosity of the interface was 0.27 , and the modulus and Poisson's ratio were $3.91 \mathrm{GPa} / 0.12$, respectively, obtained from Figure $7 \mathrm{~b}$. The CTE of the matrix was obtained from the preliminary test results, $-1.3 \times 10^{-6} / \mathrm{K}$.

\subsubsection{Study on Mechanical Properties of Fiber Rod and Fiber Bundle}

In this section, based on the microstructure characteristics and random distribution of fiber rod (bundle), FEM and Python language programming were used to generate the fiber rod (bundle) geometric model satisfying the distribution law of microstructure characteristics, and the homogenization method was used to predict the stiffness properties. Figure 8 shows the microscopic observation image and geometric model of the fiber rod. The model consists of fiber monofilament, matrix and pores. 


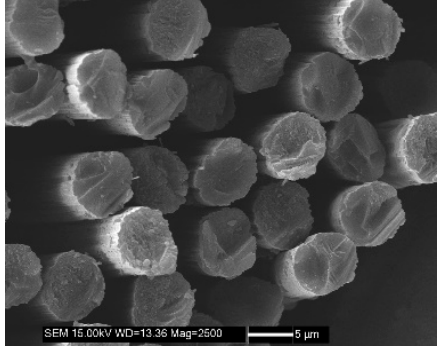

(a) Filament form

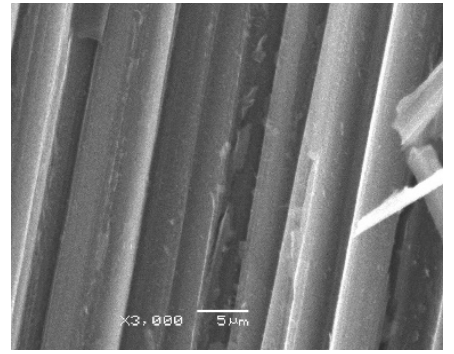

(b) lateral form

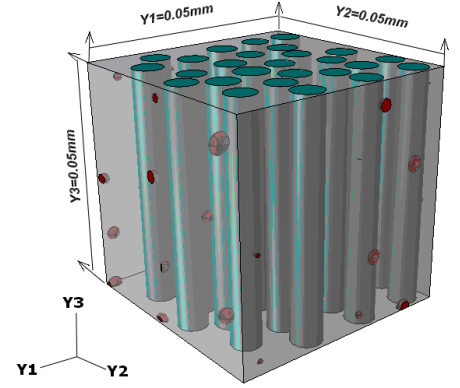

(c) geometric model

Figure 8. SEM morphology and geometric model of fiber rod (bundle).

Based on relevant experiments and combined with literature [16,17], the input parameters of fiber monofilament and matrix were determined as shown in Table 3. According to the literatures, these data were directly measured by the experiment.

Table 3. Properties of fiber and matrix in reinforcing phase.

\begin{tabular}{|c|c|c|c|c|c|c|c|}
\hline Item & $E_{x}, \mathrm{GPa}$ & $E_{y}, \mathrm{GPa}$ & $G_{x y}, \mathrm{GPa}$ & $v_{x y}$ & $v_{y z}$ & $\alpha_{\mathrm{x}}, 10^{-6} / \mathrm{K}$ & $\alpha_{\mathrm{y}}=\alpha_{\mathrm{z}}, 10^{-6} / \mathrm{K}$ \\
\hline T300 fiber & 345.1 次 $120.0 \star$ & 19.0 & 26.7 & 0.2 & 0.35 & -0.78 & 8.14 \\
\hline Matrix & 12.34 & - & - & 0.23 & - & -1.3 & -1.3 \\
\hline
\end{tabular}

Note: 㑱 represents the tensile modulus. $\star$ represents the compression modulus. Angle mark $x$ represents the fiber filaments axial direction, Angle mark $y$ and $z$ represent the transverse direction. The parameters of the matrix are the properties without pores.

The stiffness properties of the reinforcing phase were calculated by FEM. The results show that the reinforcing phase was transversally isotropic even if there are a few pores. Table 4 lists the calculation results of the enhancement phase and some experimental results in the early stage. It can be seen from the table that the prediction error of fiber rod's tensile modulus, compression modulus and shear modulus is $13.4 \%, 13.7 \%$ and $10.4 \%$ respectively, which verifies the reliability of the calculation method for the reinforcement phase. There are two reasons for the error: the error of the test system and the test method; The inaccuracy of porosity observation leads to the inaccuracy of FEM. In the subsequent calculations of the macroscopic properties of the material, the longitudinal properties of the rod were given according to the experimental values, while the shear properties and other untested parameters were given according to the predictive values.

Table 4. Stiffness properties of the enhanced phase.

\begin{tabular}{|c|c|c|c|c|c|c|c|}
\hline Item & $E_{x}, \mathrm{GPa}$ & $E_{y}, \mathrm{GPa}$ & $G_{x y}, \mathrm{GPa}$ & $v_{x y}$ & $v_{y z}$ & $\alpha_{\mathrm{x}}, 10^{-6} / \mathrm{K}$ & $\alpha_{\mathrm{y}}=\alpha_{\mathrm{z}}, 10^{-6} / \mathrm{K}$ \\
\hline $\begin{array}{l}\text { The predictive } \\
\text { value of the fiber } \\
\text { rod }\end{array}$ & 239.5 的 $85.8 \star$ & 17.37 & 20.25 & 0.11 & 0.45 & -0.94 & 5.1 \\
\hline $\begin{array}{l}\text { Test value of fiber } \\
\text { rod }\end{array}$ & $211.199 .4 \star$ & & 22.6 & 0.06 & - & - & - \\
\hline $\begin{array}{l}\text { The predictive } \\
\text { value of fiber } \\
\text { bundle }\end{array}$ & 220.8 放79.1 & 17.11 & 18.96 & 0.12 & 0.40 & -0.99 & 4.52 \\
\hline
\end{tabular}

Note: $i$ represents the tensile modulus. $\star$ represents the compression modulus. Angle mark $x$ represents the fiber rod (bundle) axial direction, Angle mark $y$ and $z$ represent the transverse direction. 


\section{Calculation Results and Analysis}

\subsection{The Calculation Results of Elastic Properties}

Periodic boundary conditions were applied to the RVE model of the axial C/C composite material to obtain its effective properties as shown in Table 5. By comparing the prediction results with the experimental results under different conditions, it can be found that the elastic modulus of the numerical prediction results is basically consistent with the experimental results, thus verifying the effectiveness of the prediction method.

Table 5. Mechanical properties of axial braided C/C composites.

\begin{tabular}{ccccccccccc}
\hline \multirow{2}{*}{ Item } & \multicolumn{3}{c}{ Elastic Modulus GPa } & \multicolumn{3}{c}{ Poisson's Ratio } & \multicolumn{3}{c}{ Shear Modulus GPa } \\
\cline { 2 - 10 } & $\boldsymbol{E}_{\boldsymbol{X}}$ & $\boldsymbol{E}_{\boldsymbol{Y}}$ & $\boldsymbol{E}_{\boldsymbol{Z}}$ & $\boldsymbol{v}_{\boldsymbol{X}}$ & $\boldsymbol{v}_{\boldsymbol{X}}$ & $\boldsymbol{v}_{\boldsymbol{}}$ & $\boldsymbol{G}_{\boldsymbol{X} \boldsymbol{y}}$ & $\boldsymbol{G}_{\boldsymbol{X}}$ & $\boldsymbol{G}_{\boldsymbol{}}$ \\
\hline Predicted value (tension) & 37.0 & 33.6 & 44.6 & 0.37 & 0.09 & 0.11 & 12.2 & 3.3 & 2.5 \\
Experimental value (tension) & 38.3 & - & 40.5 & 0.28 & 0.09 & - & 12.6 & 3.4 & - \\
Predicted value (compression) & 22.3 & 20.5 & 21.1 & 0.38 & 0.12 & 0.15 & - & - & - \\
Experimental value (compression) & 20.9 & - & 22.1 & 0.28 & 0.09 & - & - & - & - \\
\hline
\end{tabular}

Note: Angle mark $Z$ represents the fiber rod axial direction, Angle mark $X$ and $Y$ represent the transverse direction. Notice the difference between the direction sign here and the direction sign of the meso component.

\subsection{The Calculation Result of CTE}

Based on the material parameters listed in Tables 3 and 4, the energy analysis method was used to predict the CTE of the RVE model. According to previous research experience, the effect of porosity on CTE is very weak, so this paper did not study the effect of porosity on CTE. The comparison between the predicted results and the experimental results is shown in Table 6. The experimental data of thermal expansion in the table were tested by DIL 402 PC (NETZSCH, Lanzhou, China), and the sample size was $\varphi 6 \mathrm{~mm} \times 50 \mathrm{~mm}$.

Table 6. Coefficient of thermal expansion at room temperature.

\begin{tabular}{ccc}
\hline Item & $\begin{array}{c}\text { Axial Coefficient of Thermal } \\
\text { Expansion, } \mathbf{1 0}^{-\mathbf{6}} / \mathbf{K}\end{array}$ & $\begin{array}{c}\text { Radial Coefficient of Thermal } \\
\text { Expansion, 10 }\end{array}$ \\
\hline Predicted value & 0.147 & -0.477 \\
Experimental value & 0.145 & -0.50 \\
\hline
\end{tabular}

The comparison between the results of prediction and experimental results shows that the prediction method based on energy method has certain prediction accuracy. However, the predicted value in both directions is larger than the experimental value, which may be caused by the fact that the CTE of the matrix is larger than the real value.

\subsection{Effect of Braiding Spacing on Stiffness and CTE}

The stiffness and CTE of the material can be predicted by changing the center spacing of the fiber rod. The influence of braiding spacing on the tensile modulus and CTE is shown in Figure 9. With the increase of braiding spacing, the tensile modulus and CTE in axial and radial directions decrease gradually. The main reason is that the volume content of axial fiber rod and radial fiber bundle in RVE decreases while the volume fraction of matrix increases with the increase of braiding distance. Since the modulus and CTE of the matrix are lower than that of the fiber rod and fiber bundle, the increase of the volume fraction will inevitably lead to the overall decrease of the modulus and CTE [28-33]. 


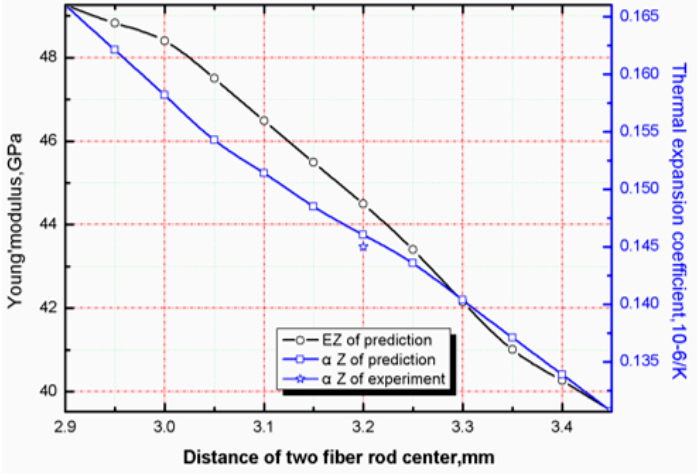

(a) Axial direction, $\mathbf{Z}$ direction

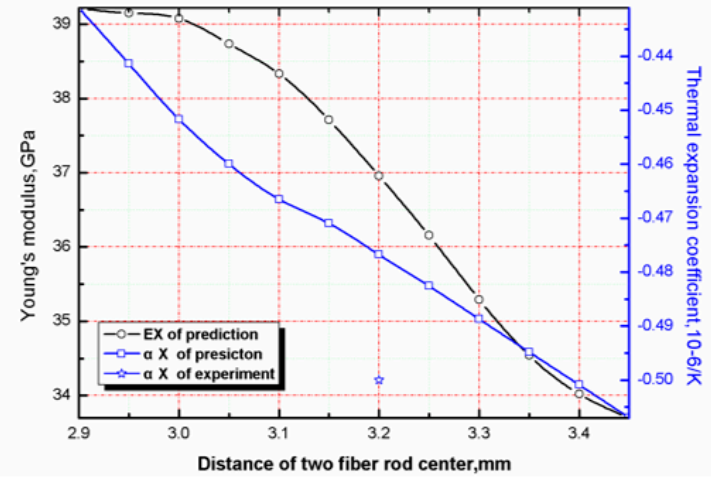

(b) Radial direction, $\mathrm{X}$ direction

Figure 9. Effect of center spacing of fiber rod on axial and radial tensile modulus and CTE.

From the perspective of the use environment of $\mathrm{C} / \mathrm{C}$ composite materials, it is necessary to reduce the modulus and CTE to reduce the thermal stress of the structural parts. Therefore, the increase of braiding spacing of materials is more in line with the requirements. However, studies have shown that the fiber volume fraction is closely related to the ablative resistance of the material. When the fiber volume fraction decreases, the ablative rate of the material increases, which is unfavorable for engineering application. Therefore, the optimization of braiding spacing of $C / C$ composites should be combined with the ablative properties of the materials. The influence of braiding spacing on ablation rate will be studied in the future.

\subsection{Effect of Fiber Rod Diameter on Stiffness and CTE}

The influence of fiber rod diameter on tensile modulus and the CTE is shown in Figure 10a. As can be seen, with the increase of fiber rod diameter, the axial tensile modulus increases significantly, while the CTE decreases significantly. This is mainly because, under the condition of constant braiding spacing, the diameter of fiber rod increases, which significantly increases the content of axial fiber in RVE. In addition, due to the higher modulus of axial fiber and the lower CTE, the axial modulus of the material increases and the CTE decreases.

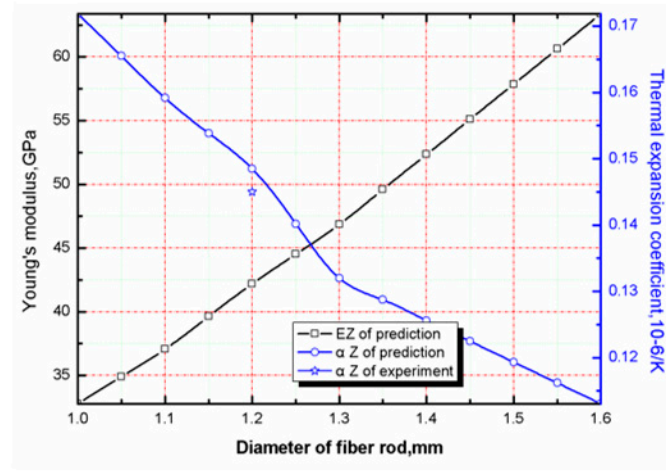

(a) Axial direction, $\mathbf{Z}$ direction

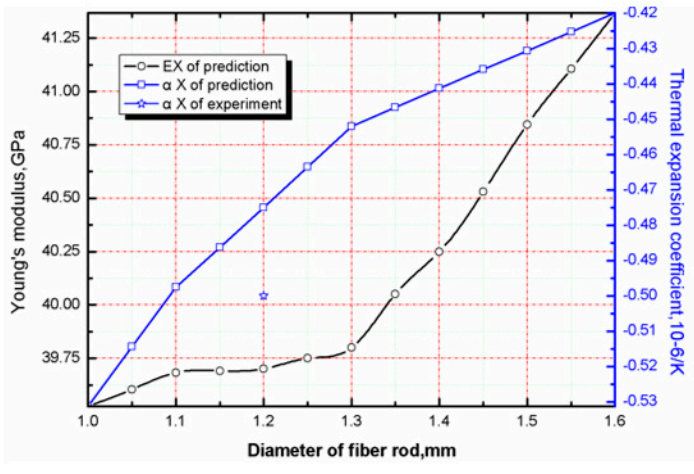

(b) Radial direction, $\mathrm{X}$ direction

Figure 10. Effect of fiber rod diameter on radial tensile modulus and CTE of axial braided C/C composites.

Radial tensile modulus and CTE are both incremental functions of fiber rod diameter, but the increment is small, as shown in Figure 10b.The reason is that for the radial tension modulus, when the braiding spacing is constant, increasing the diameter of the fiber rod will not cause the change of the volume fraction of the radial fiber bundle without changing the fiber bundle's own size, but will cause the decrease of the volume fraction of the matrix. Because the modulus of the matrix is smaller than the transverse modulus of the fiber rod, the radial tensile modulus increases. For the CTE, since 
the CTE of the matrix is smaller than transverse CTE of the fiber rod, when the volume fraction of the fiber rod increases and the volume fraction of the matrix decreases, the increase of transverse CTE of the fiber rod to the radial CTE of the material is greater than the decrease of radial CTE of the matrix. Figure 10b shows that it is appropriate for the diameter of the axial braided $\mathrm{C} / \mathrm{C}$ composite fiber rod to be around $1.26 \mathrm{~mm}$.

\section{Conclusions}

In this paper, the stiffness prediction method based on homogenization method and the CTE prediction method based on energy method were established. In the prediction of component stiffness properties, Python language was used to generate a component material model that meets the characteristics of pore microstructure randomly, so that the prediction of component material properties was more accurate. The following conclusions are obtained:

(1) The FEM was utilized for the calculation of the stiffness properties of the matrix and interface, and the results showed that the porosity was important to determine the elastic properties of the matrix and interface. It can be seen that the elastic properties of the matrix and interface change linearly with the porosity, which increases, leading to decreases of both the elastic modulus and Poisson's ratio.

(2) The stiffness performance of the reinforcing phase was calculated by FEM. The calculation results showed that the reinforcing phase manifested transversely isotropic characteristic even if there was a few of pores. It was found that the prediction precision of tensile modulus, compression modulus and shear modulus was reliable, being $13.4 \%, 13.7 \%$ and $10.4 \%$, respectively.

(3) Based on the homogenization theory and energy method combined with the RVE of axial braided C/C composite material, the macroscopic stiffness and CTE of the material were also predicted. By comparing the predicted results with the experimental results in different states, it can be concluded that the numerical predicted results are basically consistent with the experimental results, thus proving the effectiveness of the prediction method.

(4) By calculating the macroscopic stiffness performance and CTE under the conditions with different braiding spacing and fiber rod diameter, the relationship between the microstructure and macroscopic properties was evaluated. The results show that the best diameter of the axial braided C/C composite fiber rod studied in this paper is $1.26 \mathrm{~mm}$, and the optimization of braiding spacing needs to be carried out in combination with the ablative property.

(5) The advantages of the prediction method in this paper are as follows: by combining the homogenization method with FEM, the pore characteristics of the fiber rod (bundle), matrix and interface can be considered, so as to obtain the properties of mesoscopic materials accurately. As the input parameters, the stiffness prediction of the material has higher precision. The disadvantage is that the pore characteristics cannot be added into the finite element model of RVE for the joint calculation of macroscopy-mesoscopic and microscopic, which is also the focus of the follow-up research of this project.

Author Contributions: Conceptualization, C.W. and M.T.; methodology, P.C. and K.L; software, K.L.; validation, C.W., M.T. and W.T.; formal analysis, P.C.; investigation, C.W., M.T. and W.T.; resources, M.T. and W.T.; data curation, M.T. and B.L.; writing - original draft preparation, C.W. and W.T.; writing-review and editing, P.C.; visualization, M.T.; supervision, C.W.; project administration, M.T.; funding acquisition, M.T. and W.T. All authors have read and agreed to the published version of the manuscript.

Funding: The National Natural Science Foundation of China (Grant No. 51769028), The Natural Science Foundation of Qinghai Province in China (Grant No. 2017-ZJ-933Q), Beijing Institute of Structure and Environment Engineering Fund (Grant No. BQ2019001).

Acknowledgments: Thanks for the financial support from Shaanxi University of Science \& Technology.

Conflicts of Interest: The authors declare no conflict of interest. 


\section{References}

1. Hui, W.-H.; Bao, F.-T.; Wei, X.-G.; Liu, Y. Ablation performance of a 4D-braided C/C composite in a parameter-variable channel of a Laval nozzle in a solid rocket motor. New Carbon Mater. 2017, 32, 365-373. [CrossRef]

2. Wang, C.; Tang, M.; Liu, W.; Zhu, T. Study on microstructure characteristics of axially braided carbon/carbon composites based on SEM and Micro-CT. Materials 2020, 13, 1414. [CrossRef] [PubMed]

3. Gao, B.; Tang, M.; Yang, Y.; Shi, H. Mechanical experiment of 4D in-plain C/C composites. Acta Mater. Compos. Sin. 2011, 28, 245-250.

4. Rajneesh, S.; Ramesh, B.A.; Puneet, M. Finite element analysis for mechanical characterization of 4D inplane carbon/carbon composite with imperfect microstructure. Lat. Am. J. Solids Struct. 2014, 11, 170-184.

5. Li, D.S.; Yao, Q.Q.; Jiang, N.; Jiang, L. Bend properties and failure mechanism of a carbon/carbon composite with a 3D needle-punched preform at room and high temperatures. New Carbon Mater. 2016, 31, 437-444. [CrossRef]

6. Ren, J.; Zhang, Y.; Fu, Y.; Zhang, P.; Tian, S.; Zhang, L. Effects of the second phase on the microstructure and ablation resistance of HfC coating on C/C composites. Surf. Coat. Technol. 2018, 344, 250-258. [CrossRef]

7. Shan, Z.; Kang, H.; Zang, Y.; Liu, F. Microscopic structure model and finite element simulation of mechanical properties of fiber-bar composites reinforced by three dimensional weaving. Acta Mater. Compos. Sin. 2015, 32, 138-149.

8. Tan, H.; Huang, X.; Liu, L.; Guan, Y.; Chen, W. Dynamic compressive behavior of four-step three-dimensional braided composites along three directions. Int. J. Impact Eng. 2019, 134, 103366. [CrossRef]

9. Castaneda, N.; Wisner, B.; Cuadra, J.; Amini, S.; Kontsos, A. Investigation of the Z-binder role in progressive damage of 3D woven composites. Compos. Part A Appl. Sci. Manuf. 2017, 98, 76-89. [CrossRef]

10. Gerlach, R.; Siviour, R.C.; Wiegand, J.; Petrinic, N. In-plane and through-thickness properties, failure modes, damage and delamination in $3 \mathrm{D}$ woven carbon fibre composites subjected to impact loading. Compos. Sci. Technol. 2011, 72, 397-411. [CrossRef]

11. Lu, Z.; Xia, B.; Yang, Z. Investigation on the tensile properties of three-dimensional full five-directional braided composites. Comput. Mater. Sci. USA 2013, 77, 445-455. [CrossRef]

12. Zhang, P. Microstructure Modeling and Mechanical Properties Prediction of $3 d$ Needled C/C Composites; Northwestern Polytechnical University: Xi'an, China, 2016.

13. Yan, S.; Zhang, J.; Sun, B.; Guo, B. In situ measurement of strains at different locations in 3-D braided composites with FBG sensors. Compos. Struct. 2019, 230, 111527. [CrossRef]

14. Dang, H.; Zhao, Z.; Liu, P.; Zhang, C.; Tong, L.; Li, Y. A new analytical method for progressive failure analysis of two-dimensional triaxially braided composites. Compos. Sci. Technol. 2020, 186, 107936. [CrossRef]

15. Hao, W.; Huang, Z.; Zhang, L.; Zhao, G.; Luo, Y. Study on the torsion behavior of 3-D braided composite shafts. Compos. Struct. 2019, 229, 111384. [CrossRef]

16. Xu, C.-H.; Xu, D.-S.; Song, L.-Y.; Xu, K. Microstructure characterization from X-ray micro-tomography and tensile failure mechanism of 4D in-plane carbon/carbon composites. J. Solid Rocket Technol. 2013, 36, 811-815.

17. Tang, M.; Gao, B.; Yang, Y.-C.; Shi, H.-B. Mechanical properties prediction of 4D-in plane carbon/carbon composites based on homogenization method. J. Solid Rocket Technol. 2011, 34, 109-112.

18. Zhu, Z.; Qiang, H. Forecast research on micromechanical properties of $4 \mathrm{D}$ in-plane C/C composites basedon homogenization theory. J. Solid Rocket Technol. 2019, 42, 92-96.

19. Shokrieh, M.M.; Mazloomi, M.S. A new analytical model for calculation of stiffness of three-dimensional four-directional braided composites. Compos. Struct. 2012, 94, 1005-1015. [CrossRef]

20. Zhang, W.; Wang, F.; Dai, G. Topology optimal design of material microstructures using strain energy-based method. Chin. J. Aeronaut. 2007, 20, 321-328. [CrossRef]

21. Yevtushenko, A.A.; Grzes, P.; Adamowicz, A. The temperature mode of the carbon-carbon multi-disc brake in the view of the interrelations of its operating characteristics. Materials 2020, 13, 1878. [CrossRef]

22. Vignoles, G.L.; Aspa, Y.; Quintard, M. Modelling of carbon-carbon composite ablation in rocket nozzles. Compos. Sci. Technol. 2010, 70, 1303-1311. [CrossRef]

23. Liu, F.; Guan, Z.; Bian, T. Damage model for predicting shear strength of carbon/carbon composite fastener based on post-failure behavior. Compos. Struct. 2019, 221, 110864.1-110864.17. [CrossRef] 
24. Mohammed, A.S.K.; Sehitoglu, H.; Rateick, R. Interface graphitization of carbon-carbon composites by nanoindentation. Carbon 2019, 150, 425-435. [CrossRef]

25. Pimazzoni, A.; Cescon, E.; Dalla, P.M. Thermo-mechanical analysis of unidirectional carbon-carbon composite for thermal imaging diagnostic of a particle beam. Fusion Eng. Des. 2019, 146, 2457-2461. [CrossRef]

26. Li, L.; Aliabadi, M.H. Elastic property prediction and damage mechanics analysis of 3D braided composite. Theor. Appl. Fract. Mech. 2019, 104, 102338. [CrossRef]

27. Li, W.; Fang, G.; Li, W.; Liang, J.; Li, M. Role of mesoscopic features on thermochemical ablative behavior of 3D C/C braided composites. Int. J. Heat Mass Transf. 2019, 144, 118602. [CrossRef]

28. Lachaud, J.; Aspa, Y.; Vignoles, L.G. Analytical modeling of the steady state ablation of a 3D C/C composite. Int. J. Heat Mass Transf. 2008, 51, 2614-2627. [CrossRef]

29. Wang, C.G.; Xu, G.; Gong, J.L. Buckling instability of flexible joint under high pressure in solid rocket motor. Int. J. Aerosp. Eng. 2020, 2020, 10.

30. Chinnaraj, R.K.; Hong, S.M.; Kim, H.S.; Oh, P.Y. Ablation experiments of ultra-high-temperature ceramic coating on carbon-carbon composite using ICP plasma wind tunnel. Int. J. Aeronaut. Space Sci. USA 2020, in press. [CrossRef]

31. Wang, C.G.; Xu, G. The Analytical method to compute the strain on the soft PSD in double-pulse SRM. Int. J. Aerosp. Eng. 2019, 2019, 7. [CrossRef]

32. Wang, C.; Chen, C.; Ren, Q.; Chen, K. Investigation on metal diaphragm of pulse separation device in dual-pulse solid rocket motor. Proc. Inst. Mech. Eng. 2017, 231, 444-451.

33. Chowdhury, P.; Sehitoglu, H.; Rateick, R. Damage tolerance of carbon-carbon composites in aerospace application. Carbon 2018, 126, 382-393. [CrossRef]

(C) 2020 by the authors. Licensee MDPI, Basel, Switzerland. This article is an open access article distributed under the terms and conditions of the Creative Commons Attribution (CC BY) license (http://creativecommons.org/licenses/by/4.0/). 